\title{
Variations of CYP3A activity induced by antiretroviral treatment in HIV-1 infected patients
}

Received: 30 April 2004/ Accepted: 8 October 2004/Published online: 19 January 2005

(C) Springer-Verlag 2005

\begin{abstract}
Objective: To measure the in vivo variations of CYP3A activity induced by anti-HIV drugs in human immunodeficiency virus (HIV)1-positive patients. Methods: A low oral dose of midazolam (MID) $(0.075 \mathrm{mg})$ was given to the patients and the 30 -min total 1-OH midazolam (1-OHMID)/MID ratio was determined. Patients were phenotyped either before the introduction of antiretroviral treatments (control group, 90 patients) or after a variable period of antiretroviral treatment (56 patients). Twenty-one subjects underwent multiple phenotyping tests (before and during the course of the treatment).

Results: The median MID ratio was 3.51 in the control group (range 0.20-14.6). It was 5-fold higher in the group with efavirenz (28 patients; median, range: 16.0, 3.81367; $P<0.0001$ ), 13-fold lower with nelfinavir (18 patients; $0.27,0.06-36.3 ; P<0.0001)$, 17-fold lower with efavirenz + ritonavir (three patients; 0.21, 0.05-0.47; $P=0.006$ ), 50-fold lower with ritonavir (four patients; $0.07,0.06-0.17 ; P=0.0007)$, and 7-fold lower with nevirapine + (ritonavir or nelfinavir or grapefruit juice) (three patients; $0.48,0.03-1.83 ; P=0.03)$. CYP3A activity was lower in the efavirenz + ritonavir group $(P=0.01)$ and in the ritonavir group $(P=0.04)$ than in the nelfinavir group, although already strongly inhibited in the latter.
\end{abstract}

This work was supported in part by the Swiss National Research Foundation (project 3345-062092.99 and project 3200-065427.01).

J. Fellay $\cdot$ A. Telenti

Division of Infectious Diseases,

University Hospital of Lausanne, Switzerland

C. Marzolini · L. Decosterd · T. Buclin

Department of Clinical Pharmacology,

University Hospital of Lausanne, Switzerland

K. P. Golay · P. Baumann · C. B. Eap $(\bowtie)$

Unit of Biochemistry and Clinical Psychopharmacology,

Center of Psychiatric Neurosciences, Hôpital de Cery,

University Department of Adult Psychiatry,

1008 Prilly-Lausanne, Switzerland

E-mail: Chin.Eap@inst.hospvd.ch

Tel.: + 41-21-6436438

Fax: + 41-21-6436444
Conclusion: The low-dose MID phenotyping test was successfully used to measure the in vivo variations of CYP3A activity induced by antiretroviral drugs. Efavirenz strongly induces CYP3A activity, while ritonavir almost completely inhibits it. Nelfinavir strongly decreases CYP3A activity, but to a lesser extent than ritonavir. The inhibition of CYP3A by ritonavir or nelfinavir offsets the inductive effects of efavirenz or nevirapine administered concomitantly. Finally, no induction of CYP3A activity was noticeable after long-term administration of ritonavir at low dosages $(200 \mathrm{mg}$ /day b.i.d.) or of nelfinavir at standard dosages (2,500 $\mathrm{mg}$ /day b.i.d.).

Keywords CYP3A - Antiretroviral drugs · HIV-1 · Midazolam

\section{Introduction}

During previous years, significant advances have been made in the pharmacological treatment of human immunodeficiency virus (HIV) infections using highly active antiretroviral strategy. In such therapy, protease inhibitors (PIs) are routinely coadministered with other antiretroviral drugs [nucleosidic reverse transcriptase inhibitors (NRTI), non-nucleosidic reverse transcriptase inhibitors (NNRTI), and other PIs]. The rational basis for combination therapy with drugs having different mechanisms of action is to obtain an additive or synergistic inhibition of HIV replication and prevention of the emergence of drug-resistant strains. PIs and NNRTIs, unlike NRTIs, are extensively metabolized by cytochrome $P_{450}(\mathrm{CYP})$ isozymes present in the liver and in the gut wall. Among these isozymes, CYP3A, a term that in adults reflects the collective activity of CYP3A4 and CYP3A5, is the most important but other isozymes such as CYP2C9, CYP2C19, CYP2D6, and CYP2B6 also contribute [1, 2]. Nevirapine and efavirenz, two NNRTIs, induce drug-metabolizing enzymes, including 
CYP3A [1]. Most PIs are inhibitors of CYP3A, ritonavir being the most potent, and saquinavir the least [3,4]. A strong inhibition of CYP3A by ritonavir represents a potential clinical problem, considering the multiple pharmacotherapy often received by HIV-positive patients, but it is also the basis for combination treatment with other PIs such as amprenavir, indinavir, saquinavir, and lopinavir in order to increase their plasma concentrations $[5,6]$.

Interestingly, ritonavir, amprenavir and nelfinavir are both CYP3A inhibitors and inducers, and thus induce their own metabolism $[5,7,8]$. The phenomenon of concurrent inhibition and induction of drug metabolism by PIs represents a potential clinical problem as its outcome may be time dependent, a short exposure to the drug producing an inhibition that is potentially offset by induction after long-term exposure [8,9]. However, few studies, with mixed results, measured specifically CYP3A activity during the time course of antiretroviral treatment. One phenotyping study found that neither dapsone hydroxylation nor cortisol $6 \beta$ hydroxylation detected the inhibition of CYP3A by HIV-1 protease inhibitors such as ritonavir, indinavir, or amprenavir administered for 3-4 weeks to 18 patients [10]. In patients infected with HIV, the results of the erythromycin breath test were not different among a group of five patients receiving drugs reported to induce CYP3A activity (nevirapine, dexamethasone), a group of eight patients receiving drugs reported to inhibit CYP3A activity (clarithromycin, fluconazole, sertraline, fluoxetine, itraconazole), and a group of eight patients receiving drugs reported to induce and inhibit CYP3A (nevirapine, phenytoin, rifabutine, fluconazole, fluoxetine) [11]. However, nevirapine administered for 14 days significantly increased the median erythromycin breath test by $22 \%$ [11], and delavirdine decreased by $70-75 \%$ the predose erythromycin breath test values after both short- and long-term administration [12]. In a fourth study, urinary $6 \beta$-hydroxycortisol measured in HIV-positive pregnant women was comparable to that determined in the urine of healthy subjects, although the patients were all treated with PIs such as indinavir and nelfinavir [13], which are CYP3A inhibitors [4]. Finally, efavirenz significantly increased the mean erythromycin breath test result in a dose- and time-dependent manner $(55 \%$ mean induction at $400 \mathrm{mg}$ and $33 \%$ induction at $200 \mathrm{mg}$ ) in two groups of 12 healthy subjects receiving $200 \mathrm{mg}$ /day or $400 \mathrm{mg}$ /day for 10 days [14].

Some hypotheses have been put forward to tentatively explain these mixed results [10-14]. Multiple CYP isozymes play a role in the metabolism of dapsone, and it is not clear whether CYP3A is the sole metabolic pathway for the $6 \beta$-hydroxylation of cortisol [10]. The erythromycin breath test, a well-validated CYP3A probe, measures the activity of hepatic but not of intestinal CYP3A, the latter being differently regulated than the former [15]. A strong inhibition of anti-HIV drugs on intestinal, but not hepatic, CYP3A would therefore not be detected by this test. Finally, erythromycin is a substrate of the permeability glycoprotein (PGP), and how the PGP/CYP3A alliance would ultimately affect drug's metabolism [16, 17], and the results of the above-mentioned study with erythromycin as a CYP34 probe are still unclear [11, 12, 14].

The biotransformation of midazolam (MID) to its major metabolite, 1-OH midazolam (1-OHMID), is mediated by CYP3A [18]. We recently validated the use of the 1-OHMID/MID ratio determined $30 \mathrm{~min}$ after the administration of a very low oral dose of MID $(0.075 \mathrm{mg})$ as a marker of CYP3A activity [19]. The aim of the present study was to determine variations of CYP3A activity induced by various anti-HIV drugs using the low-dose MID phenotyping test and to assess the potential of this test in the follow-up of drug influences on CYP3A activity.

\section{Subjects and methods}

\section{Clinical study}

HIV-1-infected patients were recruited in a study on the influence of genetic factors on the response to antiretroviral treatment [20]. The choice of antiretroviral treatment was made independently from the study. Some preliminary results on the low-dose MID phenotyping test in HIV-1 positive patients treated with efavirenz and nelfinavir have been presented in a previous report [20], with MID ratios calculated with free (unconjugated) MID and OHMID concentrations. However, as the use of total (conjugated + unconjugated) MID and OHMID, measured after enzymatic hydrolysis of the glucuronide, enables the removal of the interindividual variability of the glucuroconjugation from the MID ratios [19, 21], samples were reanalyzed to determine total MID and OHMID concentrations. Patients declared to be free of any MID containing drugs, and it was also checked that the measured plasma levels of MID and OHMID were compatible with the administration of the low MID dose used for the phenotyping test (data not shown).

Patients were either phenotyped before the introduction of antiretroviral treatments (90 patients, 52 without comedications, 38 with comedications, none of the comedications known as being a CYP3A inhibitor or inducer) or after a variable period of antiretroviral treatment (56 patients). Among the former, 21 subjects agreed to undergo multiple phenotyping tests, i.e., before and during the course of the treatment. For these patients, the first MID phenotyping test was made on day zero, before initiation of antiretroviral treatment (which was started the same day, after the test). Second and third tests were planned after 1 day and 1 month of treatment, respectively. Data from four patients having undergone multiple phenotyping tests were excluded because of non-compliance shown by non-detectable 
blood levels of the antiretroviral agents of interest (three patients) and discrepancy between the date of the phenotyping test indicated on the tube of blood and on the case report form (one patient). Patients gave their written informed consent to participate in the study, which was approved by the ethics committee of the "Centre Hospitalier Universitaire Vaudois" (Faculty of Medicine).

\section{MID phenotyping test}

Patients were asked to drink $100 \mathrm{ml}$ water containing $0.075 \mathrm{mg}$ MID, prepared by diluting $75 \mu \mathrm{l}$ of a $1-\mathrm{mg} / \mathrm{ml}$ MID solution into $100 \mathrm{ml}$ water. The patients then drunk an additional $100 \mathrm{ml}$ water used for rinsing the glass. A blood sample was drawn $30 \mathrm{~min}$ later to determine the OHMID/MID ratio. The timing of the test was not controlled with regard to the timing of food ingestion. However, a decrease of MID absorption by food was not expected to influence the results, as a metabolite/mother substance ratio was calculated, and as the ratio obtained $30 \mathrm{~min}$ after MID intake was equivalent to the ratio obtained $1 \mathrm{~h}$ after MID ingestion [19].

Determinations of plasma concentrations of MID, 1-OHMID and of anti-HIV drugs

Plasma MID and OHMID concentrations were determined as total drug (conjugated + non-conjugated) by means of gas chromatography-negative chemical ionization mass spectrometry [22], after enzymatic hydrolysis of the glucuronic conjugates as previously described [22]. The limit of quantification was $10 \mathrm{pg} / \mathrm{ml}$ both for MID and for OHMID [22]. No interference from antiretroviral drugs was noted during the MID assay (data not shown). MID ratios were calculated as the metabolite over the parent drug blood levels multiplied by 325.8 and divided by 341.8 (the former and the latter values being the molecular weights of MID and OHMID, respectively). In the group of 90 patients phenotyped before starting the antiretroviral treatment, blood levels of total OHMID were below the limit of quantification for one patient. In the group of 56 patients phenotyped while on treatment, blood levels of MID and of total OHMID were below the limit of quantification for five patients on efavirenz, and for seven patients on PIs, respectively. For these patients, the limit of quantification of the method, i.e., $10 \mathrm{pg} / \mathrm{ml}$, was used for calculating the MID ratios.

Determination of plasma concentrations of efavirenz, ritonavir, and nelfinavir was performed using high-performance liquid chromatography (HPLC) [23]. For correlations between the MID ratios and plasma concentrations of efavirenz and nelfinavir, results were expressed as percentile distribution on the basis of our database, which included measurements of more than 4,000 drug concentrations.
Statistical analysis

The Mann-Whitney $U$-test, the Spearman correlation test and the Wilcoxon matched paired test were used when appropriate (Statistica, Release 4.5., Statsoft, Loll \& Nielsen, Hamburg, Germany). The two-sample binomial test of equal proportion was used for comparisons of frequencies. A $P$ value lower than 0.05 was considered as statistically significant.

\section{Results}

Table 1 presents a summary of clinical data. The groups of HIV-positive patients studied before the introduction of antiretroviral treatments $(n=90$, group $\mathrm{A})$ and on antiretroviral treatments $(n=56)$ did significantly differ with regard to age, time in treatment, and proportion of males. Figure 1 shows the MID ratios in the groups of patients studied before the introduction of antiretroviral treatments and on antiretroviral treatments. In the latter group, 28 patients received efavirenz (group 1), 18 patients nelfinavir (group 2), 3 patients efavirenz + ritonavir (group 3), 4 patients ritonavir (group 4), and 3 patients nevirapine (group 5; in this last group, one subject received a combination of nevirapine and nelfinavir, a second a combination of nevirapine, ritonavir, amprenavir, and lopinavir, while a third did not receive other PIs or NNRTIs but had been drinking grapefruit juice for the 3 days preceding the test). In the group of subjects phenotyped prior to antiretroviral treatment $(n=90$, group A), patients without comedications $(n=52$, group A1) did not significantly differ from patients with comedications (38 patients, group A2), with regard to gender, age, and MID ratio. Data from the whole group were therefore used as control for statistical comparisons of MID ratios with antiretroviral-treated groups.

CYP3A activity differed significantly between each group of antiretroviral-treated patients and the control group. The median MID ratios were thus 5-fold higher $(P<0.0001), 13$-fold lower $(P<0.0001), 17$-fold lower $(P=0.006), 50$-fold lower $(P=0.0007)$, and 7 -fold lower $(P=0.03)$ in the group of patients receiving efavirenz, nelfinavir, efavirenz + ritonavir, ritonavir, and nevirapine, respectively, when compared with the control group. Although already inhibited by nelfinavir, CYP3A activity was significantly lower in the efavirenz + ritonavir group $(P=0.01)$ and in the ritonavir group $(P=0.04)$ than in the nelfinavir group. Removing the data of the outlier did not change the results (data not shown). In the three patients receiving nevirapine, the highest MID ratio was measured in the patient taking grapefruit juice (1.83), the lowest in the patient with ritonavir + amprenavir + lopinavir $(0.03)$, with an intermediate value in the patient with nelfinavir $(0.48)$. In the group of 28 and 18 patients treated with efavirenz and nelfinavir, respectively, there were no significant correlations between MID ratios and blood concentrations of 
Table 1 Clinical data and 1-OH midazolam/midazolam ratios, determined using total 1-OH midazolam and midazolam (MID) concentrations, measured in different groups of HIV-1-positive patients

Group of patients $\quad$ Clinical data $\quad \begin{aligned} & \text { Statistical comparisons of MID ratios } \\ & \text { between groups }\end{aligned}$
between groups

Group A: patients before antiretroviral treatment with and without comedications

Number of patients and gender

Age (years: median; range)

MID ratio (median; range)

Group A1: patients before antiretroviral treatment without comedications

Number of patients and gender

Age (years: median; range)

MID ratio (median; range)

Group A2: patients before antiretroviral treatment with comedications

Number of patients and gender

Age (years: median; range)

MID ratio (median; range)

Group 1: patients with efavirenz $600 \mathrm{mg} /$ day q.d.

Number of patients and gender

Duration of treatment (days: median; range)

Age (years: median; range)

MID ratio (median; range)

Other antiretroviral treatment

(number of patients)

Group 2: patients with nelfinavir $2500 \mathrm{mg}$ /day b.i.d.

90 patients, 58 men

37; 19-73

$3.51 ; 0.20-14.6$

Number of patients and gender

Duration of treatment (days: median; range)

Age (years: median; range)

MID ratio (median; range)

Other antiretroviral treatment

(number of patients)

Group 3: patients with efavirenz $600 \mathrm{mg} /$ day q.d. and ritonavir $200 \mathrm{mg}$ /day b.i.d.

Number of patients and gender

Duration of treatment (days: median; range)

Age (years: median; range)

MID ratio (median; range)

Other antiretroviral treatment

(number of patients)

52 patients, 32 men

$37 ; 19-61$

3. $42 ; 0.24-14.6$

38 patients, 26 men

$38 ; 23-73$

$3.52 ; 0.20-14.5$

28 patients, 20 men

$285 ; 21-1218$

39; $20-74$

$16.0 ; 3.81-367$

Zidovudine (20), lamivudine (25),

stavudine (7), didanosine (2),

abacavir (2)

18 patients, 5 men

714; 12-1288

$42 ; 34-59$

$0.27 ; 0.06-36.3$

3 patients, 2 men

26; 24-28

$41 ; 35-51$

$0.21 ; 0.05-0.47$

Lopinavir (3), amprenavir (1),

stavudine (1), didanosine (1)

Group 4: patients with ritonavir (200 mg/day b.i.d., 2 patients; 800 /day, b.i.d., 2 patients)

Number of patients and gender

4 patients, 3 men

NS

$$
\begin{aligned}
& P<0.0001 \\
& P<0.0001 \\
& P=0.006 \\
& P=0.0007 \\
& P=0.03 \\
& \text { NS }
\end{aligned}
$$

Group A versus group 1

Group A versus group 2

Group A versus group 3

Group A versus group 4

Group A versus group 5

Group A1 versus group A2

Duration of treatment (days: median; range)

Age (years: median; range)

MID ratio (median; range)

Other antiretroviral treatment

(number of patients)

429; 28-1130

$49 ; 42-68$

$0.07 ; 0.06-0.17$

Indinavir (1), amprenavir (1),

saquinavir (2), lopinavir (1),

zidovudine (1), lamivudine (1),

stavudine (3)

Group 5: patients with nevirapine (200 mg/day b.i.d., 1 patient; $400 \mathrm{mg} /$ day, q.d., 2 patients)

Number of patients and gender

3 patients, 2 men

Duration of treatment (days: median; range)

Age (years: median; range)

MID ratio (median; range)

Other antiretroviral treatment

484; $153-1144$

54; 49-71

$0.48 ; 0.03-1.83$

(number of patients)

Nelfinavir (1), ritonavir (1), amprenavir (1), lopinavir (1),

zidovudine (1), lamivudine (2),

stavudine (1)

efavirenz $(r=0.02 ; P=0.93)$ and nelfinavir $(r=0.18$; $P=0.47$ ), respectively.

Midazolam ratios measured in patients having undergone multiple phenotyping tests are shown in Fig. 2. The last MID test, performed after a median period of treatment of 29 days (range 8-125 days), corresponds to steady-state levels for the administered antiretroviral drugs. Eleven patients received efavirenz $600 \mathrm{mg}$ /day q.d. (Fig. 2a) for a median period of
30 days (range 21-125 days). Other anti-HIV drugs prescribed to this group included zidovudine and/or lamivudine and/or stavudine and/or abacavir. Efavirenz treatment resulted in a 2.9-fold increase of median MID ratio $(P<0.005)$, but with a large variability (range of increase: $1.4-103$-fold). A significant correlation was noted between the increase of CYP3A activity and the mean CYP3A activity, i.e., (MID ratio after treatment + MID ratio at baseline $) / 2\left(r^{2}=0.48, P=0.019\right)$, i.e., a 


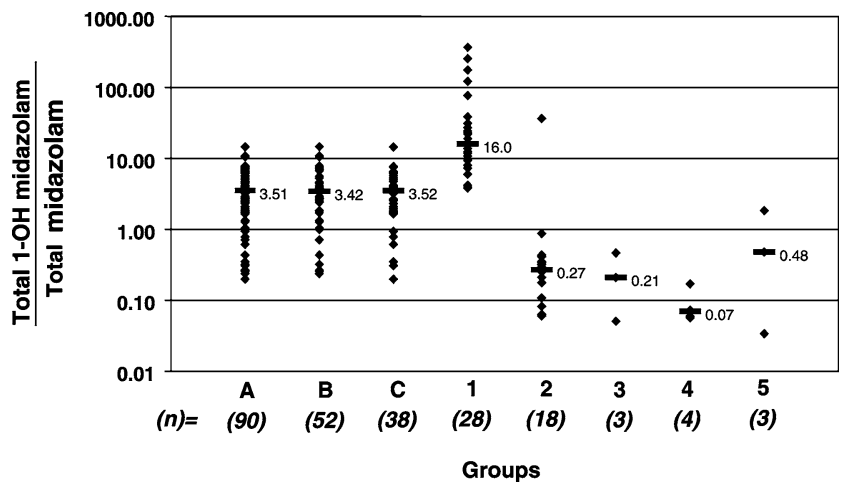

Fig. 1 Total 1-OH midazolam/midazolam ratios in different groups of HIV-1-positive patients. Group A: patients without antiretroviral treatment, with and without comedications; group A1: patients without antiretroviral treatment, without comedications; and group A2: patients without antiretroviral treatment, with comedications. Group 1: patients with efavirenz; group 2: patients with nelfinavir; group 3: patients with efavirenz + ritonavir; group 4: patients with ritonavir; and group 5: patients with nevirapine and other protease inhibitors or grapefruit juice. The median value in each group is indicated by a line

higher increase of CYP3A activity was observed in patients with a low CYP3A baseline activity. There was no correlation between the increase of CYP3A activity

Fig. 2 1-OH midazolam/midazolam (MID) ratios, determined by using total 1-OHMID and MID concentrations, in HIV-1 positive patients receiving various antiretroviral treatments (a efavirenz, b efavirenz + ritonavir, c ritonavir, d nelfinavir) measured on three occasions (first visit: before treatment, second visit: after 1 or 2 days of treatment, third visit: after at least 8 days of treatment)

a
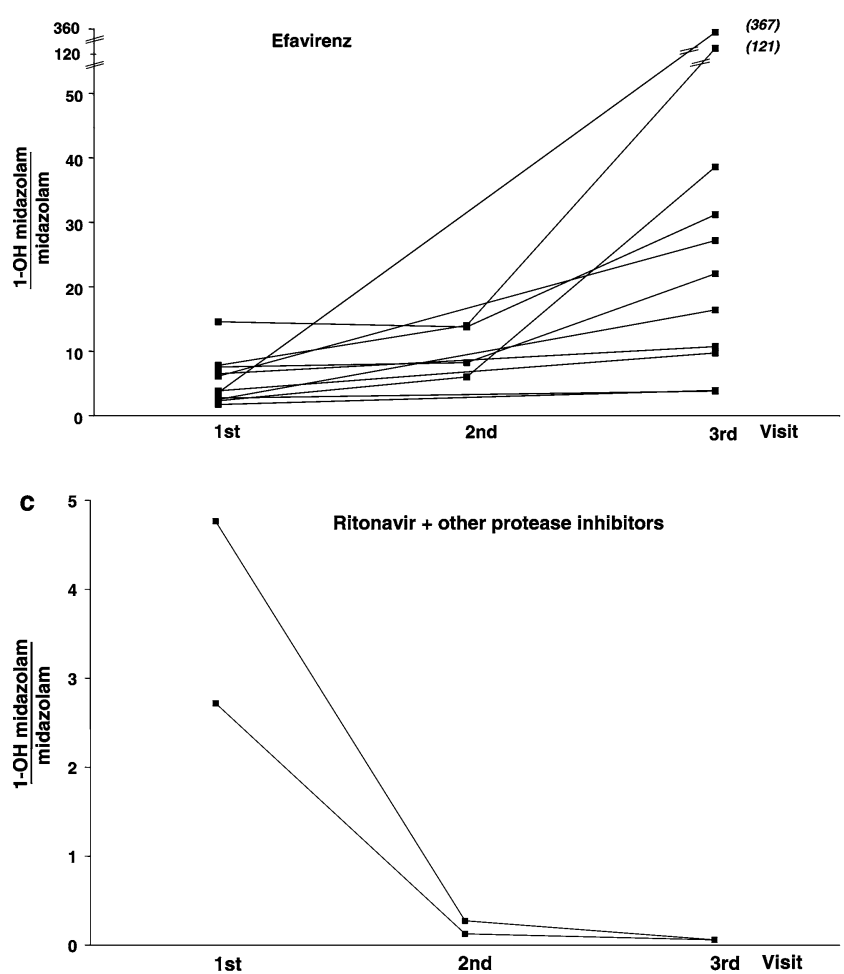

and the plasma concentrations of efavirenz $(r=-0.02$, $P=0.96)$. A trend was observed when correlating the percentage increase of CYP3A activity and the duration of treatment $(r=-0.60, P=0.051)$. Four patients were also phenotyped after the first dose: an increase of CYP3A activity (up to 2.6-fold) was measured in three of them at the second phenotyping test.

Two patients received efavirenz $600 \mathrm{mg} /$ day q.d. and ritonavir $200 \mathrm{mg} /$ day b.i.d. (Fig. 2b). These two patients received other anti-HIV drugs - as lopinavir and/or amprenavir and/or didanosine. A strong reduction in CYP3A activity was measured in these two patients, shown by a MID ratio decrease to $8 \%$ and $12 \%$ of the original value after 28 days and 42 days of treatment, respectively. Two patients receiving ritonavir without efavirenz (Fig. 2c; other anti-HIV drugs prescribed: indinavir and/or amprenavir and/or lopinavir and/or zidovudine and/or lamivudine and/or stavudine). In these two patients, an almost complete inhibition of CYP3A activity was measured after 1 month of treatment (CYP3A activity: $2 \%$ and 1\% of the baseline value). In the patient on ritonavir + efavirenz phenotyped on day 1, a 3.5-fold increase of MID ratio was observed between the test performed on day 1 and day 28, reflecting the increase in CYP3A activity. However, in the two patients on ritonavir without efavirenz, a further decrease (2.0-fold and 4.7-fold) of MID ratios was observed between the second and the third tests, showing a further decrease of CYP3A activity despite the strong inhibition already observed during the first day of treatment. Finally, in two patients receiving nelfinavir with no other PI or NNRT (other anti-HIV drugs re-
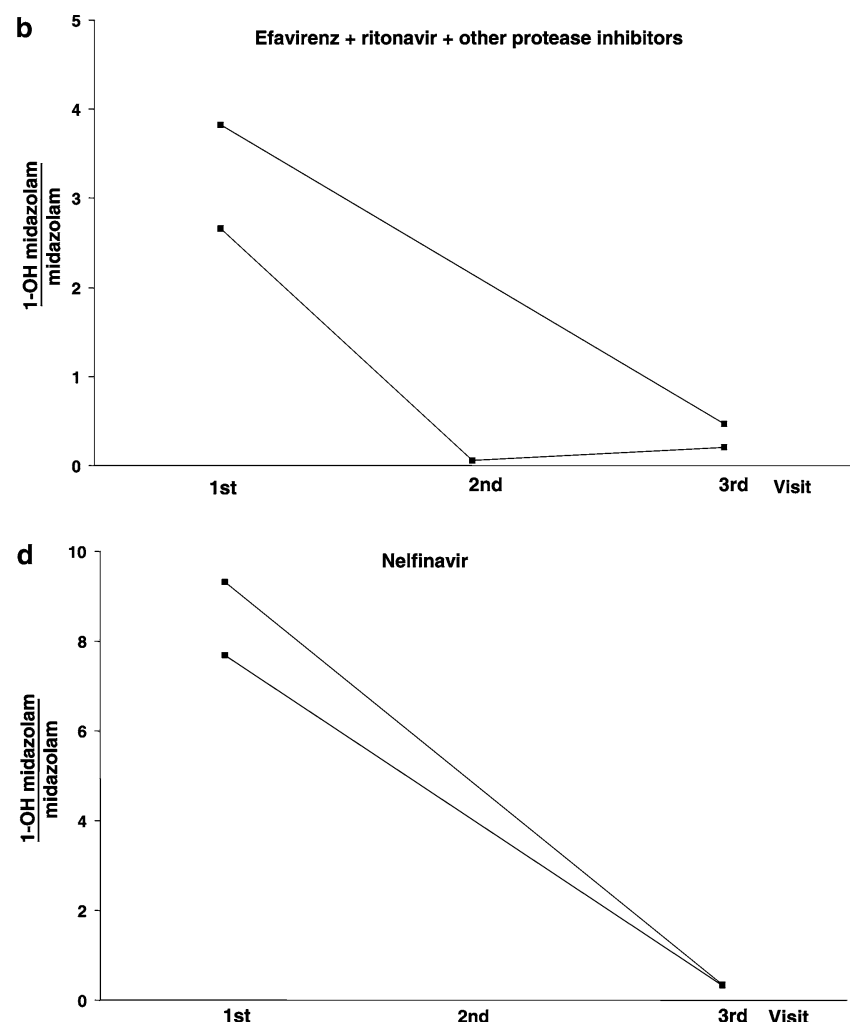
ceived: zidovudine and/or lamivudine, and/or stavudine) (Fig. 2d), a marked decrease of CYP3A activity was also noted, reflected by a MID ratio decrease to $4 \%$ of the baseline value after 8 days and 48 days of treatment.

\section{Discussion}

Drug interaction with PIs and NNRTIs is mediated through interactions with several proteins, including the PGP [24, 25] and with isoforms of the CYP family [1, 2]. With regard to the latter, an inhibition and/or induction of CYPA4 by PIs and NNRTIs is probably a core mechanism through which interactions occur. In the present study, HIV-positive patients without antiretroviral drugs (control group) and those treated with PIs (ritonavir and nelfinavir) and NNRTIs (efavirenz and nevirapine) were phenotyped with a low dose of MID. The statistical differences observed among the different groups (duration of treatment, proportion of men, age of patients) have to be discussed when comparing MID ratios among these groups. With regard to the variable duration of treatment between groups, all 56 patients can be considered in steady-state conditions, as the shortest duration of treatment was 12 days. Concerning the difference of age, the median age in the control group was 37 years, while it was 42,49 and 54 years in the nelfinavir-, ritonavir-, and nevirapinetreated groups, respectively. Ontogeny in CYP3A is well known, and small variations of CYP3A activity occur during aging. [26, 27]. However, these variations are much smaller than those observed in the present study, i.e., it is very unlikely that the abovementioned differences of age could explain the large variations of CYP3A activity observed between different groups of treatments. Finally, with regard to the high proportion of female patients observed in the nelfinavir group compared with the other groups, this should not influence CYP3A activity, as there is no statistical difference in MID clearance between men and women [28].

For the present study, MID ratios were calculated with total but also with free OHMID concentrations. The use of free MID ratios results in more overlapping values between the groups (data not shown). This difference is most probably explained by the removal of the interindividual variability linked to glucuroconjugation $[19,21]$ when using total concentrations. Thus, only total MID ratios are presented and discussed. Among HIV-positive patients without antiretroviral treatment, MID ratios were not significantly different between subjects with and without comedications. This result was expected, as none of the comedications was known to be a CYP3A inducer or inhibitor. However, the range of MID ratios appears to be wider in this group than in a group of healthy subjects without comedications (MID ratios between 2 and 11 were measured in a group of 13 healthy subjects [19]). This result is in agreement with another study that found that variability in hepatic activity of CYP3A measured using the erythromycin breath test may be greater in HIV-positive patients than in controls subjects [11]. In the present study, low MID ratios (i.e., around unity and lower) were measured in several HIV-positive patients, with or without comedications (Fig. 1). Such low ratios suggest an inhibited CYP3A activity, as values between 0.40 and 1.2 were measured in four healthy subjects treated with ketoconazole, a strong CYP3A inhibitor, $400 \mathrm{mg} /$ day for 2 days [19]. Several hypotheses can be put forward to explain the low CYP3A activity observed in some HIV-positive patients. First, although the comedications received by these patients are not known as CYP3A inhibitors, several of them are CYP3A substrates (data not shown), and they might therefore inhibit to some extent the activity of this isozyme. Second, about one-sixth of the patients contracted HIV through intravenous drug use, and the influence of a possible consumption of illicit drugs at the time of the phenotyping test is unknown. Finally, certain viral infections including HIV stimulate cytokine production, and these infections could suppress activity of CYP enzymes [11].

During initial preclinical studies, efavirenz was shown to induce its own metabolism and to increase activities of CYP3A in rats, rhesus monkeys, and humans (Bristol-Myers Squibb, unpublished data, July 2000) [29]. This has recently been confirmed by a study in healthy subjects that found a dose-dependent induction of hepatic but not intestinal CYP3A4 by efavirenz [14]. The present study also confirms the induction of CYP3A by efavirenz, as the MID ratio was significantly higher (fivefold) in the efavirenz-treated patients than in the control group. Among patients who underwent multiple phenotyping tests, the administration of efavirenz for a minimum period of 3 weeks resulted in an induction of CYP3A activity in all patients, with a median threefold increase of the MID ratio. A very large interindividual variability was observed in the increase of CYP3A activity, which can be explained, at least in part, by the baseline activity of this isozyme, i.e., a higher increase of CYP3A activity is observed in patients with a low CYP3A baseline activity. However, no clear explanation can, at present, be given for the trend toward significance when correlating the percentage increase and the number of days of treatment, with a negative correlation value (i.e., the longer is the treatment with efavirenz, the lowest is the increase in CYP3A activity). However, one can formulate the hypothesis that a reduced compliance during long-term treatment could contribute to this result or, alternatively, an autoinduction of efavirenz metabolism during long-term treatment could first contribute to lower efavirenz plasma concentrations, resulting subsequently in a decrease of CYP3A induction.

In the present study, unlike a previous report [14], no correlation was observed between the increase of CYP3A activity and the plasma concentrations of efavirenz. Presently, no explanations can be given for this discrepancy, but both studies differed by many 
factors, such as the type of subjects included (HIV positive patients versus healthy subjects), the dose of efavirenz (200 or 400 versus $600 \mathrm{mg} / \mathrm{day}$ ), and the duration of treatment (10 days versus median duration of 285 days). Also, in the above-mentioned study, the increase of erythromycin breath test was statistically significant after 4 days of efavirenz at $400 \mathrm{mg}$ /day (eight subjects), but not with efavirenz at $200 \mathrm{mg}$ /day (eight subjects) [14]. In the present study, an increase of CYP3A activity was already noticeable after 1 day of treatment with efavirenz at $600 \mathrm{mg} /$ day, i.e., after a single dose, in three of four patients. Due to the low number of subjects, this result could, however, be fortuitous, and such a rapid induction of CYP3A by the administration of $600 \mathrm{mg} /$ day efavirenz needs to be confirmed by other studies. Finally, the lack of correlation between CYP3A activity and efavirenz plasma levels determined in the present study is in agreement with the above-mentioned study, which suggested that CYP2B6, and not CYP3A, represents the most important pathway for elimination of this drug in humans [14].

Three patients received nevirapine, a CYP3A inducer [1]. However, the MID ratios suggested a slight (one patient) or a strong (two patients) inhibition of CYP3A activity. The former case can most probably be explained by the consumption of grapefruit juice, a CYP3A inhibitor [30], while the latter by the coadministration of nelfinavir or ritonavir.

Most PIs are inhibitors of CYP3A [3, 4]. This is in agreement with the present study, which clearly demonstrates a strong and significant inhibition of CYP3A activity by ritonavir and nelfinavir, when comparing data from PI-treated patients to those of the control group. Interestingly, MID ratios were significantly lower in patients receiving ritonavir $(200 \mathrm{mg} / \mathrm{day}$, as a pharmacokinetic enhancer to increase blood concentrations of other antiretroviral drugs) than in those receiving nelfinavir $(2,500 \mathrm{mg} /$ day $)$, even if efavirenz is coadministered with ritonavir. This result is in agreement with in vitro data showing that ritonavir is the most potent CYP3A inhibitor [3, 4]. In vitro studies showed that CYP3A4, CYP2D6 and CYP2C19 are the primary enzymes involved in the metabolism of nelfinavir [31]. In the present study, no correlation was observed between MID ratios and nelfinavir plasma concentrations. This could be explained by the fact that, in vivo, CYP3A does not represent the most important pathway for the elimination of this drug in humans. However, the fact that MID hydroxylation was almost completely inhibited by nelfinavir does not necessarily mean that metabolism of nelfinavir by CYP3A would also be completely inhibited.

With regard to the patients who underwent multiple phenotyping tests, two patients received efavirenz with ritonavir. In one of these patients, who was phenotyped after 1 day and after 28 days of treatment, a reduction of MID ratios was measured on day 1 relative to those measured in the baseline test, which shows that CYP3A is immediately and strongly inhibited after the first dose(s) of ritonavir. Then, the increase of MID ratio between day 1 and day 28 suggests that induction by efavirenz slightly reverses this inhibition, although inhibition by ritonavir still offsets induction by efavirenz, as shown by the approximate tenfold reduction of the MID ratios between the baseline test and the test at 1 month. The treatment of two patients with ritonavir without efavirenz resulted also in a strong decrease of CYP3A activity as early as after the first day(s) of treatment, followed by an almost complete inhibition of CYP3A activity after 1 month.

For the present study, the decision to perform a phenotyping test during the first day(s) of drug administration was motivated, in part, by our intention to determine whether a possible induction of CYP3A activity by ritonavir, or other PIs, could be detected after long-term administration of this drug, compared with a strong inhibition following the administration of the first dose(s) [9]. This dual mechanism could be a relevant clinical problem when considering the generalized use of ritonavir to increase bioavailability and, therefore, blood concentrations of other antiretroviral drugs. After the initial phase of CYP3A inhibition by ritonavir, if induction of this isozyme offsets its inhibition, a decrease of the plasma concentrations of ritonavir and of the other drugs might occur, which could lead to reduced antiviral efficacy. However, as a possible induction of CYP3A by ritonavir is probably concentration- and dose dependent, the fact that in combination treatments ritonavir is usually given at low doses, i.e., around 100 $200 \mathrm{mg} /$ day, should theoretically diminish the potential problem of CYP3A induction during treatment. In this study, data obtained in the patients receiving ritonavir show that, during a long-term treatment, if CYP3A is induced by this drug, this induction is hidden and does not offset its strong inhibitory activity, at least with such low doses. Similar results were obtained with nelfinavir at $2,500 \mathrm{mg} / \mathrm{day}$.

Midazolam is a substrate of CYP3A and probably also of PGP, although discrepant results have been published [32, 33]. As PGP is also induced by PIs [8], it has been suggested that induction of PGP by PIs could contribute to the lower drug exposure after multiple doses compared with single doses [8]. Our data showing a lack of increase of CYP3A activity after long-term administration of ritonavir and nelfinavir are in agreement with the above-mentioned study in rats treated with PIs, where no increase of hepatic CYP3A activity was measured using MID as CYP3A substrate, despite an increase of hepatic CYP3A levels [8]. The present results are also in agreement with the data we obtained in the Therapeutic Drug Monitoring Service of the University Hospital of Lausanne, which show a low intraindividual variability over time of plasma levels of PIs and NNRTIs when compliance problems are excluded and food intake standardized [34, 35]. In the latter studies, it can however be argued that, as drug levels were usually first measured after 1 month of 
treatment, induction of CYP3A would have already been complete at that time, which explains that drug determinations performed later would not differ significantly from the first blood level [34, 35]. More studies, are needed to examine the inhibiting/inducing effects of PIs toward CYP3A activity.

In summary, the present study shows that a low oral dose of MID can be used to measure the in vivo variations of CYP3A activity induced by antiretroviral drugs, despite the fact that either MID or OHMID could not be detected in a few patients. Efavirenz significantly and strongly induces CYP3A activity, while ritonavir almost completely inhibits its activity. Nelfinavir strongly decreases CYP3A activity but to a lesser extent than ritonavir. When administered together, the inhibition of CYP3A by ritonavir or nelfinavir offsets the induction properties of efavirenz or nevirapine. Finally, no increase of CYP3A activity was noticeable, due to an induction mechanism, after long-term administration of ritonavir at low dosages or of nelfinavir at standard dosages. These results are useful for assessing the activity of CYP3A, a key isozyme involved in the metabolism of a large number of drugs, in HIV-positive patients receiving a combination of antiretroviral drugs. They also support the use of low doses of ritonavir to increase bioavailability of other antiretroviral drugs, even on a long-term basis.

Acknowledgements The authors thank Prof. J. Biollaz for reviewing this manuscript, Mrs. C. Bertschi for editorial assistance, and Mrs. E. Ponce, Mrs. J. Rosselet, and Mrs. M. Gobin for bibliographic help.

\section{References}

1. Barry M, Mulcahy F, Merry C, Gibbons S, Back D (1999) Pharmacokinetics and potential interactions amongst antiretroviral agents used to treat patients with HIV infection. Clin Pharmacokinet 36:289-304

2. Malaty LI, Kuper JJ (1999) Drug interactions of HIV protease inhibitors. Drug Saf 20:147-169

3. Eagling VA, Back DJ, Barry MG (1997) Differential inhibition of cytochrome $\mathrm{P} 450$ isoforms by the protease inhibitors, ritonavir, saquinavir and indinavir. Br J Clin Pharmacol 44:190194

4. von Moltke LL, Greenblatt DJ, Grassi JM, Granda BW, Duan SX, Fogelman SM, Daily JP, Harmatz JS, Shader RI (1998) Protease inhibitors as inhibitors of human cytochromes P450: high risk associated with ritonavir. J Clin Pharmacol 38:106-111

5. Hsu A, Granneman GR, Bertz RJ (1998) Ritonavir-clinical pharmacokinetics and interactions with other anti-HIV agents [review]. Clin Pharmacokinet 35:275-291

6. Acosta EP (2002) Pharmacokinetic enhancement of protease inhibitors. J Acquir Immune Defic Syndr 29:S11-S18

7. Hsu A, Granneman GR, Witt G, Locke C, Denissen J, Molla A, Valdes J, Smith J, Erdman K, Lyons N, Niu P, Decourt JP, Fourtillan JB, Girault J, Leonard JM (1997) Multiple-dose pharmacokinetics of ritonavir in human immunodeficiency virus-infected subjects. Antimicrob Agents and Chemotherapy 41:898-905

8. Huang L, Wring S, Woolley JL, Brouwer KR, Serabjit-Singh C, Polli JW (2001) Induction of P-glycoprotein and cytochrome P450 3A by HIV protease inhibitors. Drug Metab Dispos 29:754-760
9. Greenblatt DJ, von Moltke LL, Daily JP, Harmatz JS, Shader RI (1999) Extensive impairment of triazolam and alprazolam clearance by short-term low-dose ritonavir: the clinical dilemma of concurrent inhibition and induction. J Clin Psychopharmacol 19:293-295

10. Gass RJA, Gal J, Fogle PW, Detmar-Hanna D, Gerber JG (1998) Neither dapsone hydroxylation nor cortisol 6 $\beta$-hydroxylation detects the inhibition of CYP3A4 by HIV-1 protease inhibitors. Eur J Clin Pharmacol 54:741-747

11. Slain D, Pakyz A, Israel DS, Monroe S, Polk RE (2000) Variability in activity of hepatic CYP3A4 in patients infected with HIV. Pharmacotherapy 20:898-907

12. Cheng CL, Smith DE, Carver PL, Cox SR, Watkins PB, Blake DS, Kauffman CA, Meyer KM, Amidon GL, Stetson PL (1997) Steady-state pharmacokinetics of delavirdine in HIVpositive patients: effects on erythromycin breath test. Clin Pharmacol Ther 61:531-643

13. Homma M, Beckerman K, Hayashi S, Jayewardene AL, Oka K, Gambertoglio JG, Aweeka FT (2000) Liquid chromatographic determination of urinary 6 beta-hydroxycortisol to assess cytochrome p-450 3A activity in HIV positive pregnant women. J Pharm Biomed Anal 23:629-635

14. Mouly S, Lown KS, Kornhauser D, Joseph JL, Fiske WD, Benedek IH, Watkins PB (2002) Hepatic but not intestinal CYP3A4 displays dose-dependent induction by efavirenz in humans. Clin Pharmacol Ther 72:1-9

15. Tsunoda SM, Velez RL, von Moltke LL, Greenblatt DJ (1999) Differentiation of intestinal and hepatic cytochrome P450 3A activity with use of midazolam as an in vivo probe: effect of ketoconazole. Clin Pharmacol Ther 66:461-471

16. Lan L-B, Dalton JT, Schuetz EG (2000) Mdr1 limits CYP3A metabolism in vivo. Mol Pharmacol 58:863-869

17. Benet LZ, Cummins CL (2001) The drug efflux-metabolism alliance: biochemical aspects. Adv Drug Deliv Rev 50:S3-S11

18. Wrighton SA, Thummel KE (2000) CYP3A. In: Levy RH, Thummel KE, Trager WF, Hansten PD, Eichelbaum M (eds) Metabolic drug interactions. Lippincott Williams and Wilkins, Philadelphia, pp 115-133

19. Eap CB, Buclin T, Cucchia G, Zullino D, Hustert E, Bleiber G, Powell Golay K, Aubert AC, Baumann P, Telenti A, Kerb R (2004) Oral administration of a low dose of midazolam (75 micrograms) as an in vivo probe for CYP3A activity. Eur $\mathbf{J}$ Clin Pharmacol 60:237-246

20. Fellay J, Marzolini C, Meaden ER, Back DJ, Buclin T, Chave JP, Decosterd LA, Furrer H, Opravil M, Pantaleo G, Retelska D, Ruiz L, Schinkel AH, Vernazza P, Eap CB, Telenti A (2002) Response to antiretroviral treatment in HIV-1-infected individuals with allelic variants of the multidrug resistance transporter 1: a pharmacogenetics study. Lancet 359:30-36

21. Thummel KE, Shen DD, Podoll TD, Kunze KL, Trager WF, Bacchi CE, Marsh CL, Vicar J.P., Barr CL, Perkins JD (1994) Use of midazolam as a human cytochrome $\mathrm{P} 4503 \mathrm{~A}$ probe: II. characterization of inter- and intraindividual hepatic CYP3A variability after liver transplantation. J Pharmacol Exp Ther 271:557-566

22. Eap CB, Bouchoux G, Powell Golay K, Baumann P (2004) Determination of picogram levels of midazolam, and 1-and 4hydroxymidazolam in human plasma by gas chromatographynegative chemical ionization-mass spectrometry. J Chromatogr B 802:339-345

23. Marzolini C, Telenti A, Buclin T, Biollaz J, Decosterd LA (2000) Simultaneous determination of the HIV protease inhibitors indinavir, amprenavir, saquinavir, ritonavir, nelfinavir and the non-nucleoside reverse transcriptase inhibitor efavirenz by high-performance liquid chromatography after solid-phase extraction. J Chromatogr B 740:43-58

24. Williams GC, Sinko PJ (1999) Oral absorption of the HIV protease inhibitors: a current update. Adv Drug Deliv Rev 39:211-238

25. Choo EF, Leake B, Wandel C, Inamura H, Wood AJJ, Wilkinson GR, Kim RB (2000) Pharmacological inhibition of P- 
Glycoprotein transport enhances the distribution of HIV-1 protease inhibitors into brain and testes. Drug Metab Dispos 28:655-660

26. de Wildt SN, Kearns GL, Leeder JS, van den Anker JN (1999) Cytochrome P450 3A-ontogeny and drug disposition. Clin Pharmacokinet 37:485-505

27. Sotaniemi EA, Arranto AJ, Pelkonen O, Pasanen M (1997) Age and cytochrome P450-linked drug metabolism in humans: an analysis of 226 subjects with equal histopathologic conditions. Clin Pharmacol Ther 61:331-339

28. Kashuba ADM, Bertino JS, Rocci ML, Kulawy RW, Beck DJ, Nafziger AN (1998) Quantification of 3-month intraindividual variability and the influence of sex and menstrual cycle phase on CYP3A activity as measured by phenotyping with intravenous midazolam. Clin Pharmacol Ther 64:269277

29. Marzolini C, Troillet N, Telenti A, Baumann P, Decosterd LA, Eap CB (2000) Efavirenz decreases methadone blood concentrations. AIDS 14:1291-1292

30. Bailey DG, Malcolm J, Arnold O, Spence JD (1998) Grapefruit juice-drug interactions. Br J Clin Pharmacol 46:101-110
31. Baede-van Dijk PA, Hugen PWH, Verweij-van Wissen CPWGM, Koopmans PP, Burger DM, Hekster YA (2001) Analysis of variation in plasma concentrations of nelfinavir and its active metabolite M8 in HIV-positive patients. AIDS 15:991-998

32. Kim RB, Wandel C, Leake B, Cvetkovic M, Fromm MF, Dempsey PJ, Roden MM, Belas F, Chaudhary AK, Roden DM, Wood AJJ, Wilkinson GR (1999) Interrelationship between substrates and inhibitors of human CYP3A and P-glycoprotein. Pharm Res 16:408-414

33. Tolle-Sander S, Rautio J, Wring S, Polli JW, Polli JE (2003) Midazolam exhibits characteristics of a highly permeable PGlycoprotein substrate. Pharm Res 20:757-764

34. Marzolini C, Telenti A, Decosterd LA, Greub G, Biollaz J, Buclin T (2001) Efavirenz plasma levels can predict treatment failure and central nervous system side effects in HIV-1-infected patients. AIDS 15:71-75

35. Marzolini C, Buclin T, Decosterd LA, Biollaz J, Telenti A (2001) Nelfinavir plasma levels under twice-daily and threetimes-daily regimens: high interpatient and low intrapatient variability. Ther Drug Monit 23:394-398 